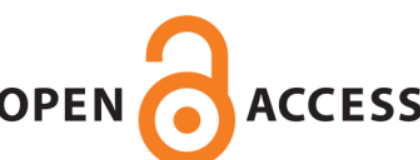

UWS Academic Portal

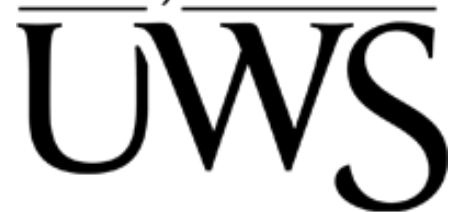

\title{
Partitioning based incremental marginalization algorithm for anonymizing missing data streams
}

Otgonbayar, Ankbayar; Pervez, Zeeshan; Dahal, Keshav

\section{Published in:}

Proceedings of the 2019 13th International Conference on Software, Knowledge, Information Management and Applications (SKIMA)

\section{DOI:}

10.1109/SKIMA47702.2019.8982399

Published: 06/02/2020

Document Version

Peer reviewed version

Link to publication on the UWS Academic Portal

Citation for published version (APA):

Otgonbayar, A., Pervez, Z., \& Dahal, K. (2020). Partitioning based incremental marginalization algorithm for anonymizing missing data streams. In Proceedings of the 2019 13th International Conference on Software, Knowledge, Information Management and Applications (SKIMA) (IEEE Proceedings). IEEE. https://doi.org/10.1109/SKIMA47702.2019.8982399

\section{General rights}

Copyright and moral rights for the publications made accessible in the UWS Academic Portal are retained by the authors and/or other copyright owners and it is a condition of accessing publications that users recognise and abide by the legal requirements associated with these rights. 
“(C) (C) 2020 IEEE. Personal use of this material is permitted. Permission from IEEE must be obtained for all other uses, in any current or future media, including reprinting/republishing this material for advertising or promotional purposes, creating new collective works, for resale or redistribution to servers or lists, or reuse of any copyrighted component of this work in other works." 


\section{Partitioning based incremental marginalization algorithm for anonymizing missing data streams}

\author{
$1^{\text {st }}$ Ankhbayar Otgonbayar \\ $A V C N, C E P S$ \\ University of the West of Scotland \\ Paisley, United Kingdom \\ Ankhbayar.Otgonbayar@uws.ac.uk
}

\author{
$2^{\text {nd }}$ Zeeshan Pervez \\ AVCN, CEPS \\ University of the West of Scotland \\ Paisley, United Kingdom \\ Zeeshan.Pervez@uws.ac.uk
}

\author{
$3^{\text {rd }}$ Keshav Dahal \\ AVCN, CEPS \\ University of the West of Scotland \\ Paisley, United Kingdom \\ Keshav.Dahal@uws.ac.uk
}

\begin{abstract}
The IoT and its applications are the inseparable part of modern world. Io $T$ is expanding into every corner of the world where internet is available. IoT data streams are utilized by many organizations for research and business. To benefit from these data streams, the data handling party must secure the individuals' privacy. The most common privacy preservation approach is data anonymization. However, IoT data provides missing data streams due to the varying device pool and preferences of individuals and unpredicted devices' malfunctions of IoT. Minimization of missingess and information loss is very important for anonymizing of missing data streams. To achieve this, we introduce IncrementalPBM (Incremental Partitioning Based Marginalization) for anonymizing missig data streams. IncrementalPBM utilizes time based sliding window for missing data stream anonymization, and it aims to control the number of $Q I D$ s for anonymization while increasing the number of tuples for anonymization. Our experiment on real dataset showed IncrementalPBM is effective and efficient for anonymizing missing data streams compared to existing missing data stream anonymization algorithm. IncrementalPBM showed significant improvement; $5 \%$ to $9 \%$ less information loss, 4500 to 6000 more number of re-use anonymization while showing comparable clustering, suppression and runtime.
\end{abstract}

Index Terms-Anonymization, Internet-Of-Things, Missing data stream, Missing value, Privacy

\section{INTRODUCTION}

The Internet-Of-Things(IoT hereafter) and it's application became the inseparable part of modern world. Constantly expanding big data-sphere is built around the world for the benefit of public and private sectors. Huge amount of sensors, actuators are embedded from everyday items to houses, big buildings thus providing a chance to detect and control precisely. Such as, government agencies, big corporations and research institutions [1] utilize the IoT data for the purpose of research and business. Many organizations utilize IoT data to improve their performance and optimize the business. For example, traffic control agencies use smart car and traffic monitoring data to optimize the traffic control, smart home data are analyzed by housing agencies to improve the house conditions [2], [3].

However, utilizing these versatile data without breaching the personal privacy of the individuals is the primary concern [4], [5]. The privacy of each individual is violated when their

978-1-7281-2741-5/19/\$31.00 @2019 IEEE identity is exposed to the malicious party, the attackers can exploit this information to learn their personal information by combining with other disclosed data [6]. Therefore, it is mandatory to sanitize IoT data upon handling [4], and the most popular data sanitization approach is anonymization [6]. This method creates privacy barrier on the data by removing or replacing information which can be utilized by malicious party to breach the privacy of individuals, therefore, ensuring the individuals' information are protected from privacy disclosure while publishing [7]-[10]. Identifier of confidential information of individual that poses privacy treat when exposed to the public is called sensitive information. For example, social security number, bank details, address and phone number. In contrast, non-sensitive information that might be exploited by malicious party to create privacy breach is called quasiidentifiers ( $Q I D$ hereafter). For example, in health-care data; age, height, gender, ethnicity and area code etc.

The anonymization replaces or removes the value of quasiidentifiers and creates uncertainty provide the privacy preservation [10]-[12]. Anonymization quality is measured by information loss which is calculated by measuring the level of uncertainty in the published information [13]. There are two types of data anonymization; dataset anonymization and data stream anonymization [14]-[18]. Dataset anonymization is performed on a previously recorded data, the main goal this type of anonymization is to minimize the information loss while publishing under user-specified privacy settings [19], [20]. On the other hand, data streams are received in a sequential order and it is processed dynamically [21]. The goal of anonymizing data stream is to publish data stream without exceeding the specified publication delay while maintaining minimum information loss [22]-[24]. However, data streams anonymization quality is measured on the trade-off between publication delay and information loss [25], [26].

Moreover, data streams must be anonymized in dynamic framework with fast and consistent publication. The most suitable technique to handle fast flowing data streams is the sliding window [14]-[18]. There are two type of sliding window; count-based [14], [16] and time-based [15], [17], [27], [28]. Count-based sliding window is size constrained, and data is anonymized and published when sliding window is full. In contrast, time-based sliding window constrained by time 
and every tuple of time-based sliding window has expiration time. Therefore, data are anonymized and published when the oldest tuple of the sliding window expiring. Researchers agreed that time-based sliding window is the most feasible solution for anonymizing data streams due to its time-bounded publication property.

The individuals of IoT possess many devices that have sensing and actuating capabilities, and those devices can be used at any time. Also, there is no globally defined usage pattern for IoT devices. Therefore, IoT produce data streams that have missing values. There are four main cause for missingness in IoT data streams; different device preference of individuals, dissimilar usage pattern, unpredictable environment condition and personal information sharing control.

Anonymization of missing data streams is very challenging [29]-[31], researchers found three major techniques to treat missingness for anonymization. There are imputation, marginalization and partitioning. Firstly, for imputation, missing values are repaired by counterfeit values calculated upon or during anonymization process [32], [33]. Secondly, missing values are ignored and treated as a $N U L L$ value [34]. Finally, for partitioning, QIDs of each tuples are used to create multiple non-missing subsets of original data, then, each subset are anonymized individually [35].

The biggest challenge of anonymizing missing data streams is to achieve minimum information loss with minimized delay while dealing with the missing values. The quality of the missing data stream anonymization is affected by the sliding window size and missingness. However, sliding window is designed to run anonymization iteration for each expiring tuples, and more tuples for the anonymization iteration results less information loss. Also, the less number of QIDs in the anonymization iteration less information loss and have minimization effect on missingess. Therefore, we propose missing data stream anonymization algorithm IncrementalPBM which aims to minimize QIDs while utilizing maximum possible tuples for the anonymization under time-based sliding window. The general idea is to utilize partitioning to minimize number of QIDs for anonymization and incrementally expand the field of anonymization QIDs for more number of tuples for anonymization iteration. More details of IncrementalPBM is explained in Section IV. Experiments on real dataset proved that IncrementalPBM is more effective on anonymizing missing data streams compared to existing algorithm, demonstrating less information loss, suppression; comparable runtime and clustering, and more number of re-using.

\section{BACKGROUND}

Data stream is received in sequential order and is to be anonymized on the fly. Therefore, researchers implemented dynamic technique called sliding window to perform data stream anonymization. There are two types of sliding window; countbased and time-based. Wang et al., [22] proposed algorithm called $S W A F$ that utilizes specialization tree. When tuple is received, it runs top-down greedy approach to find the best $K$ anonymous cluster for expiring tuple under time based sliding window. Also, in [24] Jianzhong et al., presented another specialization tree based algorithm $S K Y$. This algorithm searches the most specific specialization tree node for newly arriving tuple, then it checks if the tree node is eligible to anonymize the newly arrived tuple. Otherwise, the tuple is held until their corresponding specialization tree node accumulates enough tuples to satisfy $K$-anonymity.

Cao et al., proposed CASTLE data stream anonymization algorithm in [16]. CASTLE handles data stream under countbased sliding window. Newly arriving tuples are assigned to new or existing cluster depending on the cluster enlargement cost. The cluster enlargement is measured on the gain of information loss that is occurred when adding new tuple to the existing cluster. Moreover, to reduce anonymization cost, CASTLE utilizes re-using strategy that uses generalization information of recently published $K$-anonymous clusters to anonymize expiring tuples.

Hessam and Sylvia proposed a count-based sliding window algorithm for numeric data streams anonymization called FAANST [14]. The aim of this algorithm is to improve the data quality. Therefore, they introduced $\Delta$, information loss constraint of clusters. When sliding window is full, FAANST uses $K$-means algorithm to create clusters then it publishes $K$-anonymous clusters those having less than $\Delta$ information loss. Also, it utilizes re-using strategy to minimize the information loss. FAANST outperformed CASTLE in terms of time complexity and information loss.

Wang et al. [27], found that CASTLE [16] created few huge clusters when applied on data streams, causing frequent split operations. The merge and split operations are costly, in terms of time complexity and information loss. To address this issue, they proposed $B-C A S T L E$, they set a threshold on cluster size and applying correlation distance to select merging clusters. $B$-CASTLE showed higher quality anonymization in shorter time.

Guo et al., [15] proposed time based sliding window algorithm FADS to resolve the issues of CASTLE. They found overload of clusters in CASTLE. Also, they discussed that complex merge and split functions of CASTLE are not important for data stream anonymization since the size of cluster is defined by $K$. They solved these issues by utilizing time-based sliding window, exploiting re-usable clusters in the memory, and by using $K N N$ for anonymization iteration to publish $K$-anonymous cluster. Adorenke et al., [36] mentioned that the high information loss can occur when data stream is intermittent. They proposed an adaptive buffer resizing anonymization scheme for resource constrained environment to anonymize streaming crime data. They predict the data arrival rate to resize the buffer to minimize the information loss. For the calculation of data arrival rate they utilized Poisson probability model [37] by assuming the data streams as a sequence of events occurred in a fixed time interval.

Missing value is the one of the most challenging topic for data analytics [29], [30], [33], [34]. There are three main problems that missing data causes: creates substantial amount of information bias, makes data handling and analysis 
formidable, and inefficiency [38]. Imputation, marginalization and partitioning approaches are predominantly used to address the aforementioned issues.

Imputation: Imputation is the well-known method for repairing missing values in statistical analysis. In this method, pre-calculated counterfeit values replaces the missingness of the data stream. Imputation is classified into two types: single imputation [39], [40] and multiple imputation [41], [42]. Imputation fixes the missing values of the data, however,it amplifies the uncertainty of anonymized data creating more information loss. If the imputation amount of cluster is less compared to its size, then anonymization with imputation results more secure privacy preservation while repairing the missing values.

Marginalization: In marginalization, missing values are treated as NULL values, then, utilized as normal QID value [35]. The main issue of the marginalization based approaches are the excessive amount of missing values of published clusters. However, marginalization is the most feasible solution if moderation of missingness is considered in the publication. Marginalization does not add any values to the original data and it makes published data easier to analyze [34].

Partitioning: Dataset with missing values can be divided to several subsets with no missing values, and each subset can be processed by traditional anonymization methods. However, partitioning based approaches are not cost efficient when dataset have an excessive volume of missingness in comparison to its size [18]. Nevertheless, this technique publishes clusters without missing values while ensuring the privacy preservation. Ciglic et al., proposed partitioning based anonymization approach ANON [35] for dataset containing $N U L L$ values, along with three $N U L L$ value matching scheme.

\section{PRELIMINARIES}

In tradition data streams, each tuple have same QIDs; whereas, missing data stream might contain tuples that have one ore more missing values. In the following we define the fundamentals of missing data stream and it's anonymization.

Definition 1 (Tuple of IoT data) Tuple of IoT data is defined as: $t\left(i d_{t}, Q_{t}, t s_{t}\right)$ - where $i d_{t}$ is the identity of an individual, $Q_{t}=\left\{q_{1}, q_{2}, \ldots, q_{m}\right\}$ is a set of QIDs of a tuple, and $t s_{t}$ is a time-stamp of the tuple arrival.

Definition 2 (Missing data stream) Let $Q$ be the QID's of the data stream, where $Q=\left\{q_{1}, q_{2}, \ldots, q_{n}\right\}$.The missing data streams are defined as: $S\left(i d, Q_{t}, t s\right)$ where id is the individual's identity, $Q_{t}$ is the subset of $Q\left(Q_{t} \subseteq Q\right)$ that describes a receiving tuple, and ts is the time-stamp of the tuple.

Definition 3 ( $K$-Anonymous cluster) Let $C\left(Q_{c}\right)$ be a cluster $C$ generated out of missing data stream $S$. If the $C\left(Q_{c}\right)$ contains not less than $K$ number of identities in its composition, then, $C\left(Q_{c}\right)$ is a $K$-anonymous cluster.
Definition 4 (Partition on $Q_{p}$ ) Let $P$ be a set of tuples that shares exact same QIDs set $P\left(Q_{p}\right)=\left\{t_{1}\left(\right.\right.$ pid $\left._{1}, Q_{p}, t s_{1}\right)$, $t_{2}\left(\right.$ pid $\left._{2}, Q_{p}, t s_{2}\right), \ldots, t_{z}\left(\right.$ pid $\left.\left._{z}, Q_{p}, t s_{z}\right)\right\}$, then $P$ is a partition created on $Q_{p}$.

Definition 5 (Distance between two tuples) Let $t_{1}$ (pid, $Q_{1}$ ) and $t_{2}\left(\right.$ pid, $\left.Q_{2}\right)$ be the tuple of missing data stream $S$. The distance of $t_{1}$ and $t_{2}$ is calculated on the common QIDs of both tuples.

$$
\begin{gathered}
\text { Distance }\left(t_{1}, t_{2}\right)=\frac{\sum_{q_{i} \in\left|Q_{1} \cap Q_{2}\right|} d_{i}\left(q_{i}\right)}{\left|Q_{1} \cap Q_{2}\right|} \\
d_{i}\left(q_{i}\right)= \begin{cases}\frac{\left|r_{i .1}-r_{i .2}\right|}{\left|R_{i . u}-R_{i . l}\right|} & \text { if } q_{i} \text { is numerical } \\
\frac{\mid \text { leaves }\left(H_{i}\right) \mid-1}{\mid \text { leaves }\left(D G H_{i}\right) \mid-1} & \text { if } q_{i} \text { is categorical }\end{cases}
\end{gathered}
$$

Where $r_{i .1}\left(r_{i .2}\right)$ is the value of $t_{1} . q_{i}\left(t_{2} . q_{i}\right)$ if $q_{i}$ is a numeric attribute, $H_{i}$ is the lowest common ancestor of $t_{1} . q_{i}\left(t_{2} . q_{i}\right)$ with respect to $D G H_{i}$.

Clusters generated from missing data streams can contain tuples with different composition of QIDs. Cluster generalization of missing data streams is not similar to traditional cluster generalization. We define the following cluster generalization for such clusters.

Definition 6 (Cluster generalization) Let $G_{j}^{*}\left(g_{1}, g_{2}, \ldots, g_{m}\right)$ be the generalization of cluster $C\left(Q_{j}\right)$. Following calculations are utilized to find the generalization of each $Q I D$ of $Q_{j}$ for generalization.

1) $g_{i}=\left[r_{i . \min }, r_{i . \max }\right]$, where $r_{i . \min }\left(r_{i . \max }\right)$ is the $\min (\max )$ value of $q_{i}$ in cluster $C$. If $q_{i}$ is a numerical.

2) $g_{i}=H_{\text {i.lowest }}$ where $H_{i . l o w e s t}$ is the lowest common ancestor of the $q_{i}$ values of the cluster $C$. If $q_{i}$ is a categorical.

Definition 7 (Information loss of tuple) The information loss of generalizing a tuple $t\left(\right.$ pid, $\left.Q_{t}\right)$ to $G_{t}\left(g_{1}, g_{2}, \ldots, g_{m}\right)$ is defined as follows:

$$
\operatorname{InfoLoss}\left(t, G_{t}\right)=\frac{1}{\left|G_{t}\right|}\left(\sum_{q_{i} \in Q_{t}} \operatorname{Loss}\left(q_{i}\right)\right)
$$

Where Loss $\left(q_{i}\right)$ is the attribute information loss $q_{i}$ caused by the attribute generalization $g_{i}$.

$$
\operatorname{Loss}\left(q_{i}\right)= \begin{cases}\frac{r_{i . u}-r_{i . l}}{R_{i} u-R_{i . l}} & \text { if } g_{i} \in\left[r_{i . l}, r_{i . u}\right] \\ \frac{\mid \text { leaves }\left(H_{i}\right) \mid-1}{\mid \text { leaves }\left(D G H_{i}\right) \mid-1} & \text { if } g_{i}=H\end{cases}
$$

Where $\left[r_{i . l}, r_{i . u}\right]$ is the numeric domain of a numeric attribute $q_{i}$, and $D G H_{i}$ is the domain generalization hierarchy $(D G H)$ of a categorical attribute $q_{i}, \mid$ leaves $\left(H_{i}\right) \mid$ and $\mid$ leaves $\left(D G H_{i}\right) \mid$ represents the size of a tree rooted on $H_{i}$ and $\mathrm{DGH}_{i}$ respectively. 
Definition 8 (Average information loss) The average information loss for anonymization of the first $N$ tuples of missing data stream defined as follows:

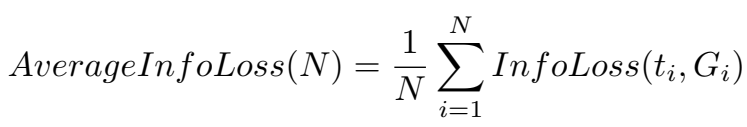

\section{INCREMENTAL PARTITIONING BASED MARGINALIZATION}

The details of IncrementalPBM are explained in Algorithm 1. IncrementalPBM has four parameters; $S$-missing data stream, $K$-anonymity degree, $\delta$-time based sliding window contraint and $\omega$ is the time contraint for re-using $K$ anonymous clusters. Firstly, IncrementalPBM reads tuple $t$ from $S$, then, depending on its QID composition, $t$ is inserted into partition in $S e t_{p}$, or new partition (see definition 4) is created on $t$ and added to $\operatorname{Set}_{p}$. Set $t_{p}$ acts as the buffer in IncrementalPBM().

When tuples is expiring, it removes all expired $K$-anonymous re-usable clusters from $S_{e t} t_{k c}$, then, IncrementalPBM () checks the size of the buffer. If the size of the buffer is greater or equal to $K$ it calls procedure AnonymizationPBM(t) to publication, otherwise, SuppressAnonymization $(t)$ is called. The details of procedure Anonymization $P B M(t)$ and SupppressAnonymization $(t)$ are shown in Algorithm 2 and Algorithm 3 respectively.

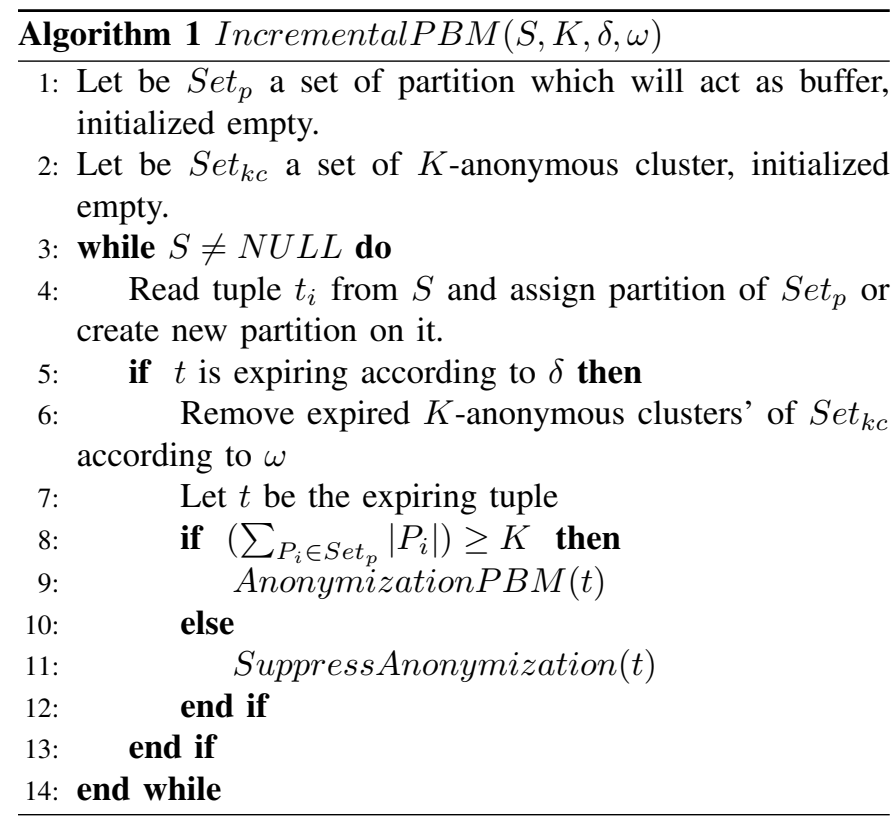

Anonymization PBM $(t)$ is the most important part of our algorithm. Firstly, it tries to find $K$-anonymous cluster $C_{\text {min }}$ from $S_{e t} t_{k c}$ which covers $t$ with minimum information loss. If $C_{\min }$ is found, then, simply, $t$ is published with re-use. If $t$ is not published by re-use, it creates temporary partitions set $S_{p u b}$ and inserts all the partitions that are defined on the QIDs that can be covered by QID of expiring tuple $t$, and anonymization iteration QID is set as $t$ 's QID set. Therefore, number of tuples for $K N N$ is increased without extending the QID set of anonymization iteration. Then, it checks the size of $S_{p u b}$ to ensure enough tuple is accumulated for $K N N$. If there is not enough tuple for $K N N$ it finds most similar and biggest partition using Jaccard's similarity [43] to expand QID set of anonymization iteration. Following the incremental expansion of QID set of anonymization iteration, more partitions are added to $S_{p u b}$ for anonymization. This incremental process continues until $S_{\text {pub }}$ collects enough tuple for $K N N$. When the incremental accumulation complete, it runs $K N N$ and forms new cluster $C_{n e w}$ and published it. Also, $C_{\text {new }}$ is added to $\operatorname{Set}_{k c}$.

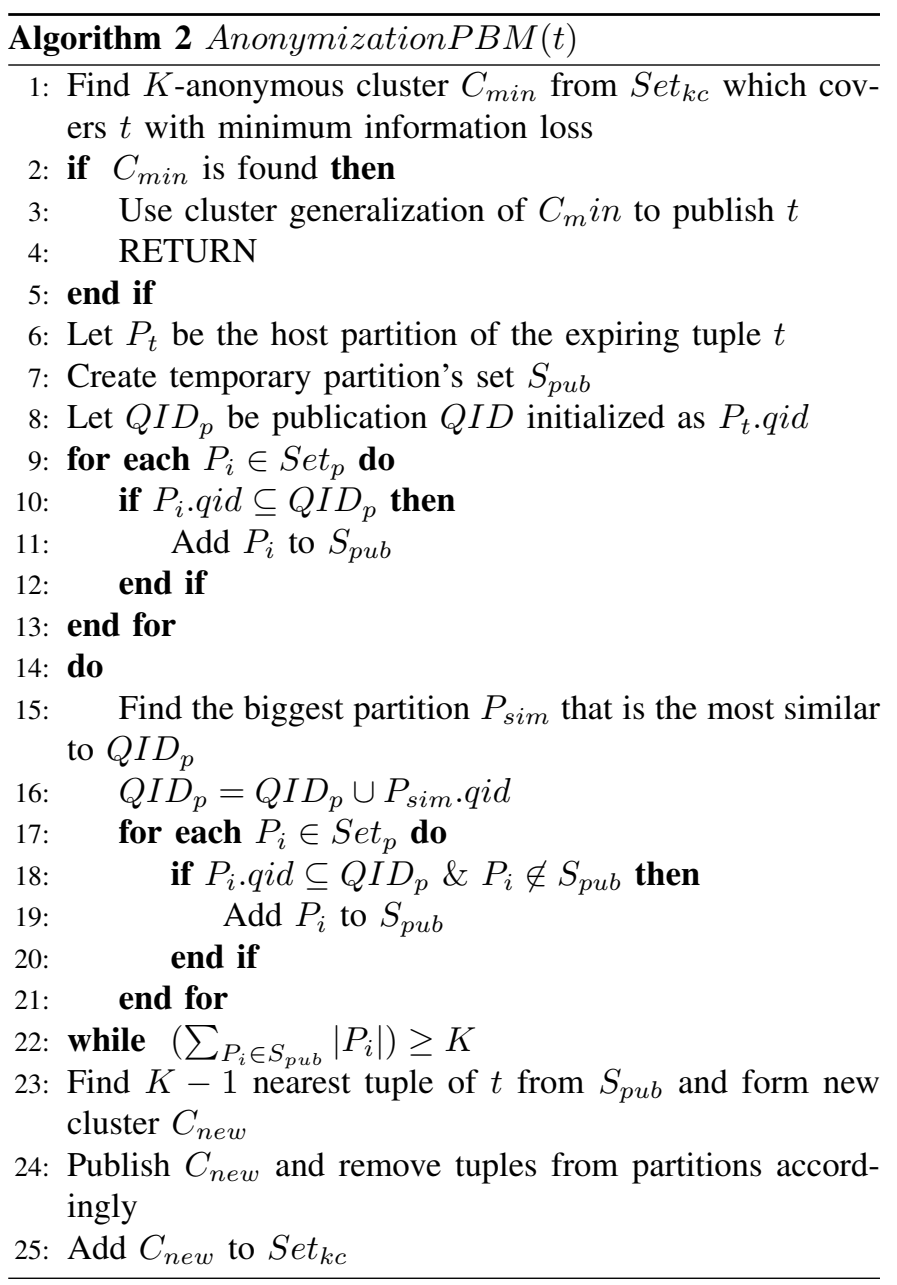

SuppressAnonymization $(t)$ is called when data streams is ended or interrupted. It tries to find $K$-anonymous cluster $C_{\text {min }}$ from $S e t_{k} c$ which covers $t$ with minimum information loss (see definition 3). If such $K$-anonymous cluster is found, then it publishes $t$ with the generalization information of $C_{m i n}$. Otherwise, tuple $t$ is suppressed and published.

\section{EXPERIMENTAL EVALUATION}

To evaluate the performance of IncrementalPBM, we compared it with $K$-VARP [18]. The $K$-VARP is the most recent algorithm for anonymizing missing data streams. We 


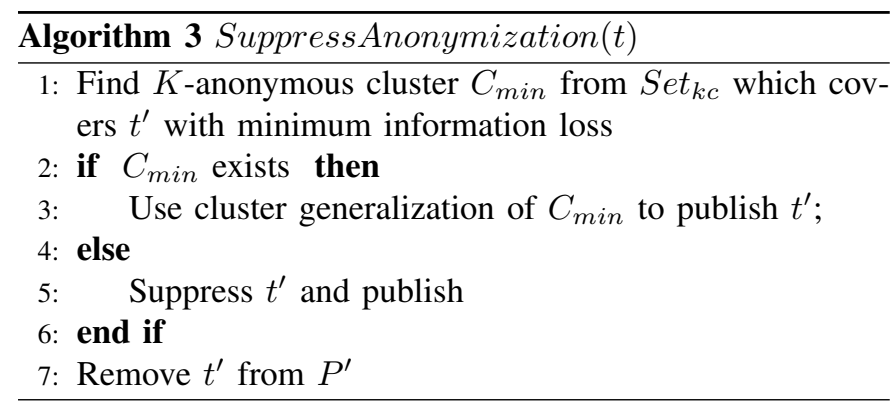

TABLE I

PARAMETERS OF ALGORITHMS

\begin{tabular}{|l|l|}
\hline Algorithm & Parameters \\
\hline$K$-VARP & $K=50, \delta=2000, \omega=2000, R=0.2$ \\
\hline IncrementalPBM & $K=50, \delta=2000, \omega=2000$ \\
\hline
\end{tabular}

used famous dataset Adult ${ }^{1}$ to evaluate the anonymization algorithms [14]-[16], [18], [27]. Adult dataset has fourteen $Q I D$ s containing eight categorical and six numeric attributes which are: education, marital-status, work-class, occupation, relationship, race, gender, country and age, final-weight, education-number, capital-gain, capital-loss. The generalization hierarchy of eight categorical attributes are defined in [13], and summary of Adult dataset is described in [18]. We used 30000 tuples of Adult dataset for the experiment, and missing values are added to imitate the missing data stream. To simulate consistent flow of data stream each tuple is retrieved from the dataset with the delay of $500 \mathrm{~ms}$. Both algorithms are implemented in Java. The experiments are performed on a PC with Intel ${ }^{\circledR}$ Core ${ }^{\mathrm{TM}}$ i7-6700HQ CPU with 16GB RAM and Windows 10x64 with JDK8.0.

The parameter for the experiments shown in Table I, where $K$ is the $K$-anonymity, $\delta$ is the time constraint of the sliding window, $\omega$ is the time constraint re-usable $K$-anonymous clusters and $R$ is the $R$-likeness of $K-V A R P$.

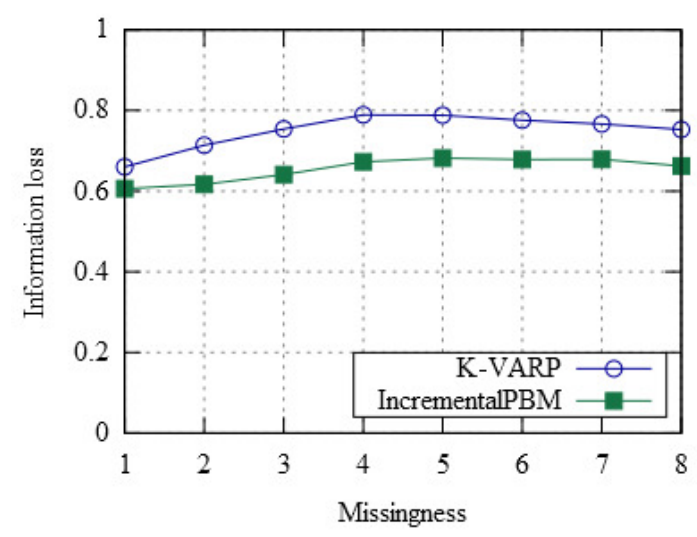

Fig. 1. Average information loss of IncrementalPBM and $K$-VARP

The average information loss on both IncrementalPBM and $K$-VARP is illustrated in Fig. 1. The average information loss

\footnotetext{
${ }^{1}$ https://archive.ics.uci.edu/ml/datasets/adult
}

is calculated using equation (5). Overall, IncrementalPBM demonstrated around 5\% to 9\% percent less information loss compared to $K$-VARP. Both algorithm showed increase of information loss when missingness of the data is increased from 1-missing to 4-missing. However, IncrementalPBM performent more consistent and efficient anonymization in terms of information loss. The less information of IncrementalPBM is caused by the incremental partitioning based marginalization which increased the number of tuples for $K N N$ while minimizing the number of QIDs for anonymization.

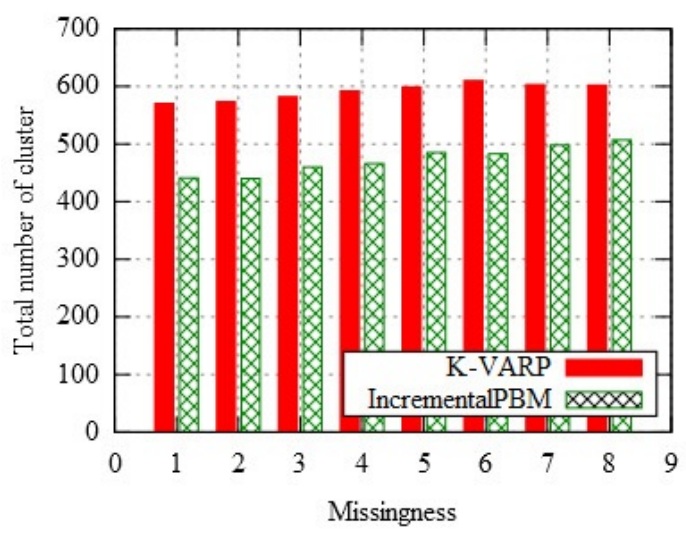

Fig. 2. Number of Clustering of IncrementalPBM and $K$-VARP

Fig. 2 demonstrated the number of clusters created for IncrementalPBM and $K$-VARP. $K$-VARP published approximately $30 \%$ more clusters compared to the IncrementalPBM. Nevertheless, we can see gradual increase on number of clusters when missingness of data is increasing. $K$-VARP utilizes less number of partitions for single anonymization iteration compared to IncrementalPBM. Therefore, less number of small partitions are appeared for $K$-VARP and this caused less number of clustering for IncrementalPBM.

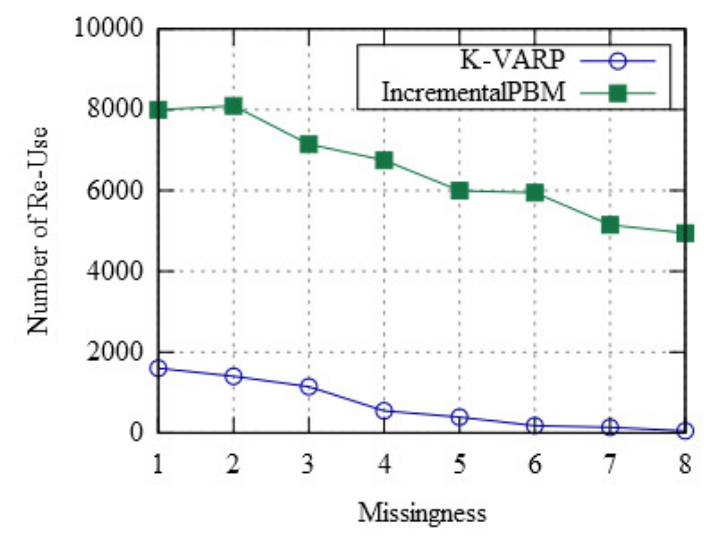

Fig. 3. Number of Re-Use of IncrementalPBM and $K$-VARP

The number of re-use anonymization of IncrementalPBM and $K$-VARP are shown in Fig. 3. The difference of reuse anonymization is very large, IncrementalPBM re-use anonymized more than 4500 to 6000 tuples compared to $K$ - 
$V A R P$. IncrementalPBM uses less time to prepare tuples for $K N N$. To merge partitions in $K-V A R P$ calculates distance of every tuples of partitions, in contrast, IncrementalPBM uses partitions' QID and size for partition accumulation. Also, IncrementalPBM prioritizes re-use before clustering, and this resulted big amount of re-use anonymization. Fig. 4 depicts the number of suppression for both IncrementalPBM and $K$ $V A R P$. Number of suppressions for both algorithms are not substantial compared to the size of the test dataset. $K$-VARP suppressed one to fourteen tuples, whereas, IncrementalPBM suppressed one to six tuples. Substantial number of re-using occured for IncrementalPBM, and reasonably fit number of clustering occured on $K$-VARP. Therefore, minimal suppression is expected for the both algorithms.

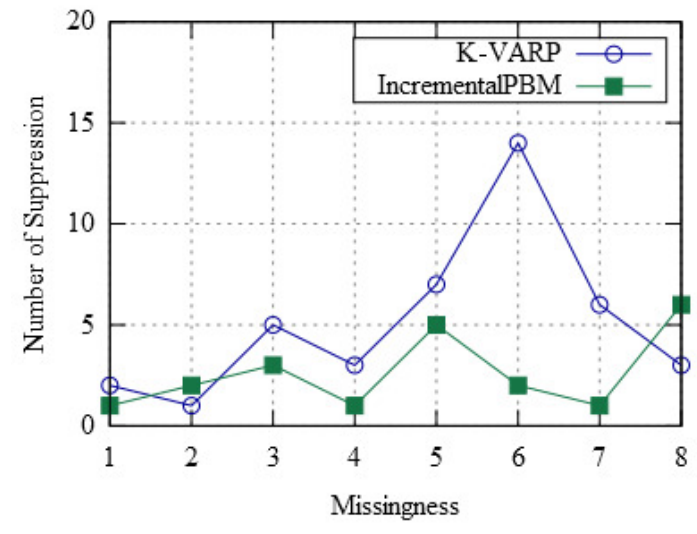

Fig. 4. Number of Suppression of IncrementalPBM and $K$-VARP

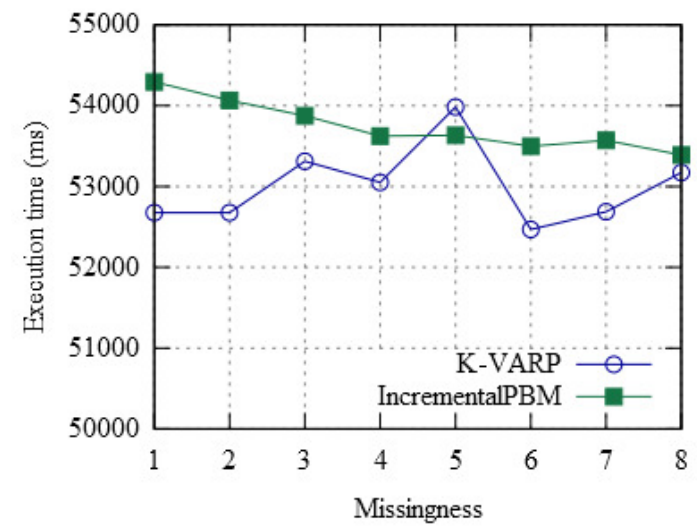

Fig. 5. Runtime of IncrementalPBM and $K$-VARP

The runtime of algorithms are illustrated on Fig. 5. Overall, IncrementalPBM showed very steady runtime for all the experiments, run time of IncrementalPBM is gradually decreased from $54400 \mathrm{~ms}$ to $53400 \mathrm{~ms}$ when missingess of the data is increased. On the other hand, K-VARP showed unstable runtime. Also, when missingness is less, then, $K$ $V A R P$ spent noticeable less time for anonymization compared IncrementalPBM. Overall, average runtime difference of both algorithm is approximately $500 \mathrm{~ms}$ which is relatively short time compared to the total runtime.

\section{CONCLUSION}

In this paper, we presented an algorithm IncrementalPBM for anonymizing missing data streams. IncrementalPBM can be utilized to process missing data streams generated from IoT environment. For example, condition monitoring of buildings, smart parking and smart homes. The incremental partitioning based marginalization helps to control the number of QIDs for anonymization while increasing the number of tuples for anonymization. Compared to existing algorithm $K$-VARP, IncrementalPBM showed considerable improvement, $5 \%$ to $9 \%$ less information loss, 4500 to 6000 more number of re-use anonymization while showing comparable number of clusters created, tuples suppressed, and runtime. For future work, we will concentrate on the optimization of clustering of missing values in IoT system data streams. Finally, we will work on the development of adaptive partitioning based marginalization algorithm for anonymizing missing data streams using artificial intelligence techniques.

\section{ACKNOWLEDGEMENT}

The first author likes to acknowledge the support provided by the EU Erasmus Mundus project gLINK (552099-EM-12014-1-UK-ERA) to pursue this research at the University of the West of Scotland, UK.

\section{REFERENCES}

[1] M. Oppitz and P. Tomsu, "Internet of things," in Inventing the Cloud Century. Springer, 2018, pp. 435-469.

[2] P. Krishnan, "Design of collision detection system for smart car using li-fi and ultrasonic sensor," IEEE Transactions on Vehicular Technology, 2018.

[3] W. Alsafery, B. Alturki, S. Reiff-Marganiec, and K. Jambi, "Smart car parking system solution for the internet of things in smart cities," in 2018 1st International Conference on Computer Applications \& Information Security (ICCAIS). IEEE, 2018, pp. 1-5.

[4] J. Gubbi, R. Buyya, S. Marusic, and M. Palaniswami, "Internet of things (iot): A vision, architectural elements, and future directions," Future generation computer systems, vol. 29, no. 7, pp. 1645-1660, 2013.

[5] X. Li, R. Lu, X. Liang, X. Shen, J. Chen, and X. Lin, "Smart community: an internet of things application," IEEE Communications Magazine, vol. 49, no. 11, 2011

[6] J. Tang, Y. Cui, Q. Li, K. Ren, J. Liu, and R. Buyya, "Ensuring security and privacy preservation for cloud data services," ACM Computing Surveys (CSUR), vol. 49, no. 1, p. 13, 2016

[7] L. Sweeney, "k-anonymity: A model for protecting privacy," International Journal of Uncertainty, Fuzziness and Knowledge-Based Systems, vol. 10, no. 05, pp. 557-570, 2002.

[8] A. Machanavajjhala, J. Gehrke, D. Kifer, and M. Venkitasubramaniam, " \ell-diversity: Privacy beyond $\backslash$ kappa-anonymity," in null. IEEE, 2006 p. 24

[9] T. M. Truta and B. Vinay, "Privacy protection: p-sensitive k-anonymity property," in null. IEEE, 2006, p. 94.

[10] N. Li, T. Li, and S. Venkatasubramanian, "t-closeness: Privacy beyond k-anonymity and 1-diversity," in Data Engineering, 2007. ICDE 2007. IEEE 23rd International Conference on. IEEE, 2007, pp. 106-115.

[11] K. LeFevre, D. J. DeWitt, and R. Ramakrishnan, "Incognito: Efficient full-domain k-anonymity," in Proceedings of the 2005 ACM SIGMOD international conference on Management of data. ACM, 2005, pp. 49-60.

[12] X. Zhang, C. Liu, S. Nepal, and J. Chen, "An efficient quasi-identifier index based approach for privacy preservation over incremental data sets on cloud," Journal of Computer and System Sciences, vol. 79, no. 5, pp. $542-555,2013$ 
[13] V. S. Iyengar, "Transforming data to satisfy privacy constraints," in Proceedings of the eighth ACM SIGKDD international conference on Knowledge discovery and data mining. ACM, 2002, pp. 279-288.

[14] H. Zakerzadeh and S. L. Osborn, "Faanst: fast anonymizing algorithm for numerical streaming data," in Data privacy management and autonomous spontaneous security. Springer, 2011, pp. 36-50.

[15] K. Guo and Q. Zhang, "Fast clustering-based anonymization approaches with time constraints for data streams," Knowledge-Based Systems, vol. 46, pp. 95-108, 2013.

[16] J. Cao, B. Carminati, E. Ferrari, and K.-L. Tan, "Castle: Continuously anonymizing data streams," IEEE Transactions on Dependable and Secure Computing, vol. 8, no. 3, pp. 337-352, 2011.

[17] H. Zakerzadeh and S. L. Osborn, "Delay-sensitive approaches for anonymizing numerical streaming data," International journal of information security, vol. 12, no. 5, pp. 423-437, 2013.

[18] A. Otgonbayar, Z. Pervez, K. Dahal, and S. Eager, "K-varp: Kanonymity for varied data streams via partitioning," Information Sciences, vol. 467, pp. 238-255, 2018.

[19] J. Han, J. Yu, Y. Mo, J. Lu, and H. Liu, "Mage: A semantics retaining k-anonymization method for mixed data," Knowledge-Based Systems, vol. 55, pp. 75-86, 2014.

[20] A. Oganian and J. Domingo-Ferrer, "Local synthesis for disclosure limitation that satisfies probabilistic k-anonymity criterion." Transactions on Data Privacy, vol. 10, no. 1, pp. 61-81, 2017.

[21] S. Kim, M. K. Sung, and Y. D. Chung, "A framework to preserve the privacy of electronic health data streams," Journal of biomedical informatics, vol. 50, pp. 95-106, 2014.

[22] W. Wang, J. Li, C. Ai, and Y. Li, "Privacy protection on sliding window of data streams," in Collaborative Computing: Networking, Applications and Worksharing, 2007. CollaborateCom 2007. International Conference on. IEEE, 2007, pp. 213-221.

[23] J. Wang, C. Deng, and X. Li, "Two privacy-preserving approaches for publishing transactional data streams," IEEE Access, vol. 6, pp. 23648 23658,2018

[24] J. Li, B. C. Ooi, and W. Wang, "Anonymizing streaming data for privacy protection," 2008

[25] K. Al-Hussaeni, B. C. Fung, and W. K. Cheung, "Privacy-preserving trajectory stream publishing," Data \& Knowledge Engineering, vol. 94, pp. 89-109, 2014

[26] M. Al-Zobbi, S. Shahrestani, and C. Ruan, "Experimenting sensitivitybased anonymization framework in apache spark," Journal of Big Data, vol. 5, no. 1, p. 38, 2018.

[27] P. Wang, J. Lu, L. Zhao, and J. Yang, "B-castle: An efficient publishing algorithm for k-anonymizing data streams," in Intelligent Systems (GCIS), 2010 Second WRI Global Congress on, vol. 2. IEEE, 2010, pp. $132-136$.

[28] A. Otgonbayar, Z. Pervez, and K. Dahal, "Toward anonymizing iot data streams via partitioning," in Mobile Ad Hoc and Sensor Systems (MASS), 2016 IEEE 13th International Conference on. IEEE, 2016, pp. 331336.

[29] X. Yan, W. Xiong, L. Hu, F. Wang, and K. Zhao, "Missing value imputation based on gaussian mixture model for the internet of things," Mathematical Problems in Engineering, vol. 2015, 2015.

[30] J. W. Graham, "Multiple imputation with norm 2.03," in Missing data. Springer, 2012, pp. 73-94.

[31] B. Fekade, T. Maksymyuk, M. Kyryk, and M. Jo, "Probabilistic recovery of incomplete sensed data in iot," IEEE Internet of Things Journal, vol. 5, no. 4, pp. 2282-2292, 2018.

[32] C.-F. Tsai, M.-L. Li, and W.-C. Lin, "A class center based approach for missing value imputation," Knowledge-Based Systems, vol. 151, pp. 124-135, 2018.

[33] Z.-g. Liu, Q. Pan, J. Dezert, and A. Martin, "Adaptive imputation of missing values for incomplete pattern classification," Pattern Recognition, vol. 52, pp. 85-95, 2016.

[34] K. Wagstaff, "Clustering with missing values: No imputation required," in Classification, Clustering, and Data Mining Applications. Springer, 2004, pp. 649-658.

[35] M. Ciglic, J. Eder, and C. Koncilia, "Anonymization of data sets with null values," in Transactions on Large-Scale Data-and KnowledgeCentered Systems XXIV. Springer, 2016, pp. 193-220.

[36] A. B. Sakpere and A. V. Kayem, "On anonymizing streaming crime data: A solution approach for resource constrained environments," in IFIP International Summer School on Privacy and Identity Management. Springer, 2017, pp. 170-186.
[37] S. Li, "Poisson process with fuzzy rates," Fuzzy Optimization and Decision Making, vol. 9, no. 3, pp. 289-305, 2010.

[38] J. Barnard and X.-L. Meng, "Applications of multiple imputation in medical studies: from aids to nhanes," Statistical methods in medical research, vol. 8, no. 1, pp. 17-36, 1999.

[39] E.-L. Silva-Ramírez, R. Pino-Mejías, and M. López-Coello, "Single imputation with multilayer perceptron and multiple imputation combining multilayer perceptron and k-nearest neighbours for monotone patterns," Applied Soft Computing, vol. 29, pp. 65-74, 2015.

[40] Z. Zhang, "Missing data imputation: focusing on single imputation," Annals of translational medicine, vol. 4, no. 1, 2016.

[41] P. Li, E. A. Stuart, and D. B. Allison, "Multiple imputation: a flexible tool for handling missing data," Jama, vol. 314, no. 18, pp. 1966-1967, 2015.

[42] S. Van Buuren, Flexible imputation of missing data. Chapman and Hall/CRC, 2018.

[43] S. Kosub, "A note on the triangle inequality for the jaccard distance," Pattern Recognition Letters, vol. 120, pp. 36-38, 2019. 